\title{
Rejection of atmospheric muons in KM3NeT/ORCA
}

\section{Luigi Antonio Fusco* for the KM3NeT Collaboration}

Dipartimento di Fisica e Astronomia dell'Università di Bologna, Viale Berti-Pichat 6/2, 40127, Bologna, Italy.

INFN - Sezione di Bologna, Viale Berti-Pichat 6/2, 40127, Bologna, Italy.

E-mail: Ifusco@bo.infn.it

KM3NeT is a future research infrastructure hosting the next-generation underwater neutrino observatory in the Mediterranean Sea. Within KM3NeT, the ORCA detector will be devoted to the measurement of the neutrino mass hierarchy, by investigating matter-induced effects in the oscillation pattern of atmospheric neutrinos.

The main background for this search is given by atmospheric muons coming from cosmic-ray air showers. Misreconstructed downward-going atmospheric muons can mimic upward going events induced by neutrinos and represent a major difficulty in this high precision measurement. Monte Carlo simulations have been used to develop a rejection strategy for these events, based only on the output of event reconstruction algorithms without requiring a larger surrounding detector or the use of a part of the instrumented volume for veto purposes.

A percent-level contamination of atmospheric muons in the final selected sample can be obtained without significant deterioration of the neutrino selection efficiency.

The 34th International Cosmic Ray Conference,

30 July- 6 August, 2015

The Hague, The Netherlands

\footnotetext{
* Speaker.
} 


\section{Introduction}

KM3NeT [1] is the next generation neutrino telescope being built in the Mediterranean Sea. It will comprise several thousand Digital Optical Modules (DOMs) [2], pressure resistant glass spheres housing 31 3-inch photomultiplier tubes (PMTs). DOMs will be distributed along vertical strings [3], each holding 18 of them. 115 strings arranged together will constitute a detector building block. Prototypes for the KM3NeT detector are already being tested under the sea [4].

In contrast to KM3NeT/ARCA [5] which, with a large spacing between DOMs (36 m) and strings (90 m) and made of two building blocks, will be devoted to high energy astrophysics [5], KM3NeT/ORCA, a much denser apparatus having a shorter inter-DOM distance and strings spaced in average by $20 \mathrm{~m}$, made by one building block, will address low energy neutrino physics [6]. Optimisation studies on the vertical spacing between ORCA DOMs are ongoing. For the study reported in these proceedings, the distance between DOMs on a string is $6 \mathrm{~m}$.

The determination of the Neutrino Mass Hierarchy (NMH) has been recentrly suggested to be possible in the context of future low threshold - large mass Čerenkov detectors under water or ice [7]. Measuring the Mikheyev-Smirnov-Wolfenstein (MSW) effect in atmospheric neutrino oscillations through matter $[8,9]$, the possibility of identifying the NMH is being investigated both for the under-water, i.e. for KM3NeT/ORCA, and under-ice (PINGU - at the South Pole) options.

The sensitivity of these of detectors to the $\mathrm{NMH}$ is highest in the energy range where Earth traversing neutrinos undergo resonant flavor oscillations, i.e. for $3<\mathrm{E}_{v}<10 \mathrm{GeV}$ [10]. Low energy neutrinos are considered the signal for this search. The most abundant background for the observation of atmospheric neutrinos is due to wrongly reconstructed atmospheric muons, mimicking upward going neutrino induced muons.

Since the difference between the two possible mass hierarchy scenarios is small, the contamination from atmospheric muon background must be low and the loss of selected tracks in this region is to be minimized. Good energy and angular resolution is required for selected events. The performance of reconstruction algorithms achieved for track and shower events are reported in $[11,12]$.

\section{Muon rejection strategy}

The main background in the observation of atmospheric neutrinos is given by atmospheric muon bundles from extensive air showers. Locating the detector under some kilometer of rock, water or ice reduces this background significantly, but not enough to have a pure neutrino signal. However, only neutrinos arrive from below the detector, as they can traverse the Earth because of their low cross section with matter at the relevant energies. On the other hand, the atmospheric muon flux is completely absorbed above $13 \mathrm{~km}$ of water equivalent of traversed material: atmospheric muon bundles arrive at the detector only from above.

The signal induced on PMTs by down-going atmospheric muons can in any case mimic that of neutrino induced upward-going tracks. The strategy for rejecting these wrongly reconstructed atmospheric muons in KM3NeT/ORCA will be described here. Since for ORCA there will not be a larger surrounding detector to provide a veto for atmospheric muons (as IceCube for PINGU), everything must be based on the output of reconstruction algorithms. 


\subsection{Reconstruction quality parameters}

Atmospheric muon bundles arriving at the detector are generated with the MUPAGE software [13], a fast event generator using parametrisation of the energy, zenith and multiplicity distributions of atmospheric muons under water or ice [14]. Cherenkov radiation is then generated and particles and light propagated through the detector, taking into account the water properties. Light detection on the DOMs is finally simulated together with the data acquisition system - simulated data after the entire Monte Carlo chain are produced in a format that is analogous to what will be present in real data.

Simulated events are reconstructed using the track reconstruction strategy described in [11]. The algorithm is based on a likelihood maximisation procedure taking into account the time of detected hits and the expected Cherenkov light yield from a straight muon track. The maximum of the likelihood function $L_{\max }$ divided by the number of degrees of freedom of the fit

$$
\Lambda=\frac{\log \left(L_{\max }\right)}{N_{h i t}-5}
$$

is a parameter defined to assess the goodness of the track fit. Under the assumption of a multivariate Gaussian distribution of the likelihood around its maximum, the error covariance is obtained, from which the angular error estimate $\beta$ is computed.

The $\Lambda$ distribution for all upward going reconstructed events is shown in figure 1 . Wrongly reconstructed atmospheric muons can be rejected cutting on the $\Lambda$ parameter. However, this simple rejection strategy does not allow to avoid a strong loss of signal neutrinos, even combining the $\Lambda$ cut with a $\beta$ selection. This improved muon rejection lowers in any case the neutrino detection efficiency, especially at low energies where the highest NMH significance is reached.

\subsection{Track starting point}

Along with the $\Lambda$ and $\beta$ parameters the reconstruction algorithm provides an estimation of the muon track starting point by back-tracking the selected hits along the muon path. While this quantity is a crude estimation of the neutrino interaction vertex, in the case of atmospheric muons it does not represent a physical quantity, but rather a "pseudo-vertex" underlining these wrongly reconstructed events. The position of the track starting point can be used, indeed, to select and identify atmospheric muons as shown in figures 2 ( $\mathrm{X}$ and $\mathrm{Y}$ coordinates with respect to the detector centre of gravity) and 3 ( $\mathrm{R}$ and $\mathrm{Z}$ coordinates). In the case of atmospheric muons the track starting point is located well outside the instrumented volume, while neutrinos have their starting point much closer to the apparatus.

Figure 1 shows the effect of a selection on the radial distance from the detector centre of the reconstructed track starting point on the cumulative $\Lambda$ distribution for atmospheric muons and low energy neutrinos. At a given cut on the value of the $\Lambda$ parameter, e.g. $\Lambda>-5$, the number of surviving muons is reduced by more than a factor 500 with respect to the event rates detected when no radial selection is applied. This holds almost uniformly as a function of the $\Lambda$ selection, with some improvement, as one would expect, for higher quality tracks $(\Lambda>-4)$ the number of surviving atmospheric muons is reduced by 3 orders of magnitude for a radius cut at $107 \mathrm{~m}$. However, moving to a stricter quality selection decreases significantly the number of surviving signal events; 


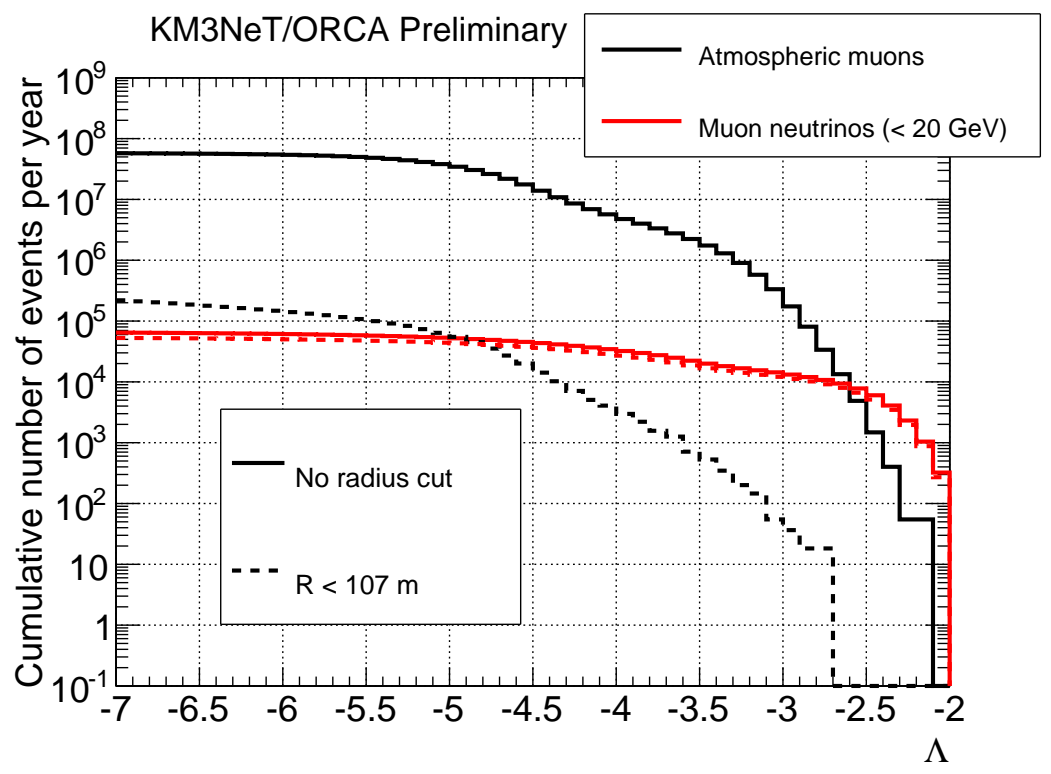

Figure 1: Cumulative $\Lambda$ distribution for events reconstructed as upward-going. Wrongly reconstructed atmospheric muons in black, muon neutrinos is red. The dashed lines show the $\Lambda$ distribution when a cut on the radial distance of the track starting point with respect to the detector centre of gravity is applied ( $\mathrm{R}<$ $107 \mathrm{~m}$, i.e. the detector radius), compared to the situation when no radial selection is done (solid lines). The atmospheric muon background is reduced by almost 3 orders of magnitude.

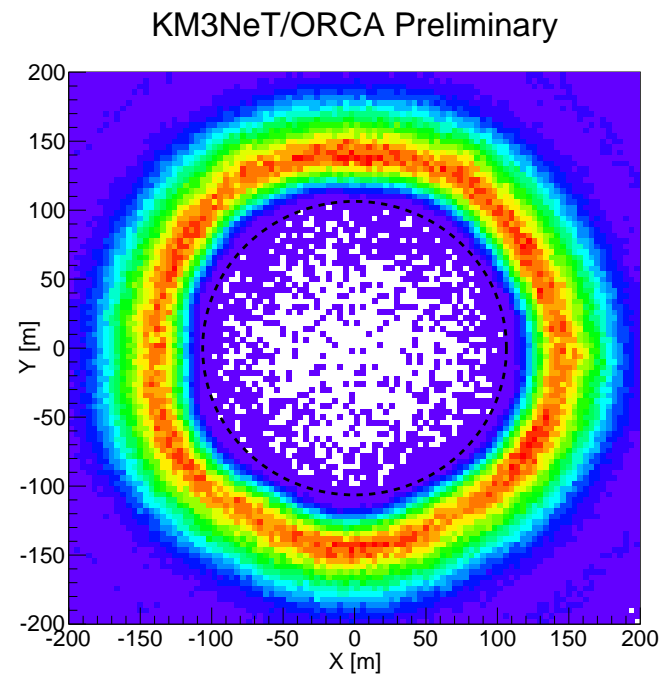

(a) Atmospheric muons

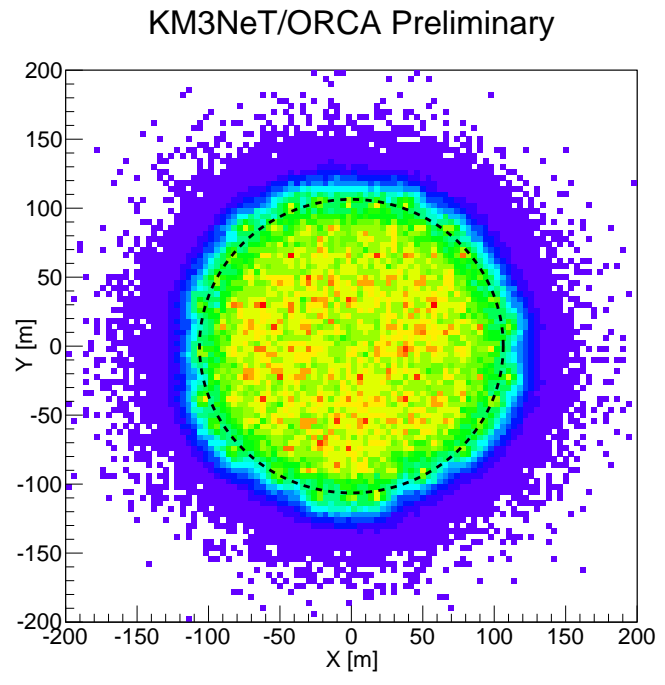

(b) Atmospheric muon neutrinos, below $20 \mathrm{GeV}$

Figure 2: $x-y$ distribution of the track starting points for events reconstructed as upward going: a) is for atmospheric muons, b) for muon neutrinos $\left(\mathrm{E}_{v}<20 \mathrm{GeV}\right)$. A quality cut on the track reconstruction is applied, namely $\Lambda>-5$. The dashed circle shows the instrumented volume of KM3NeT/ORCA building block. 


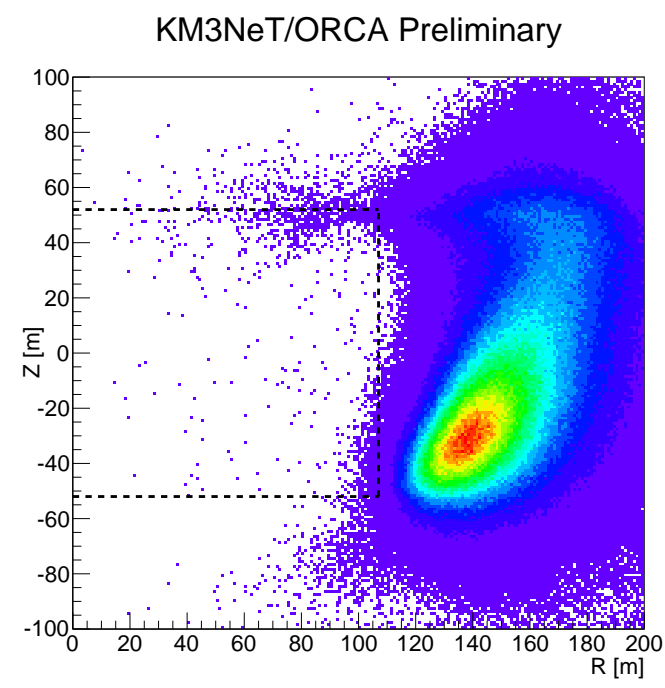

(a) Atmospheric muons

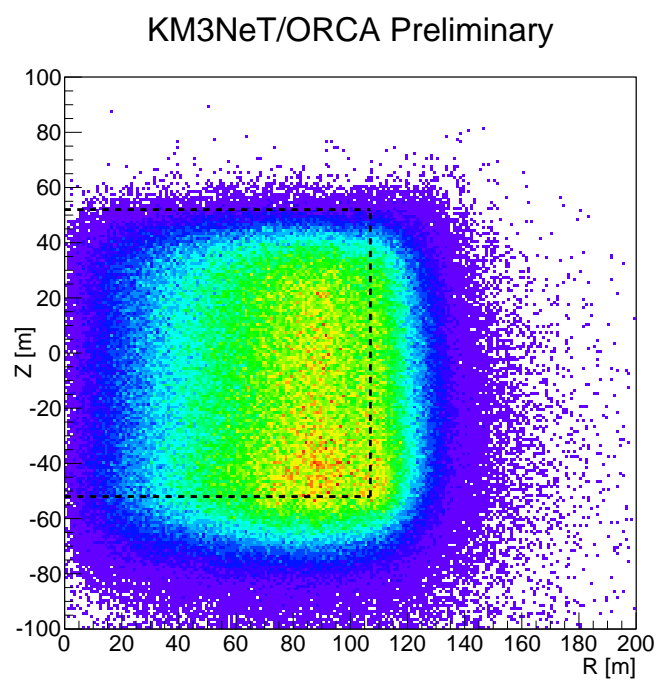

(b) Atmospheric muon neutrinos, below $20 \mathrm{GeV}$

Figure 3: R-z distribution of the track starting points for events reconstructed as upward-going: a) is for atmospheric muons, b) for muon neutrinos $\left(\mathrm{E}_{v}<20 \mathrm{GeV}\right)$. A quality cut on the track reconstruction is applied, namely $\Lambda>-5$. The dashed rectangle shows the instrumented volume of a KM3NeT/ORCA building block.

a percent contamination of atmospheric muons can be reached only losing most of signal events. A more accurate rejection tool must be used to improve the selection efficiency.

\subsection{Boosted Decision Tree technique}

The ROOT Toolkit for MultiVariate Analysis (TMVA) [15] has been used to perform a further rejection of atmospheric muons on the basis of the output of the track reconstruction algorithm. The reconstructed zenith angle, the $\Lambda$ and $\beta$ parameters and the $\mathrm{R}$ and $\mathrm{Z}$ coordinates of the reconstructed track starting point are used as input for the Boosted Decision Tree (BDT) method. This machine learning technique is trained on Monte Carlo samples of low energy neutrinos and of atmospheric muons arriving at the detector, reconstructed as upward-going and with the track starting point within the radius of the instrumented volume.

The result of the application of the BDT to the signal and background samples is shown in figure 4. A selection cut on this output quantity can provide the final sample selection, optimised on the signal to noise ratio. The contamination from atmospheric muons in the final sample can be reduced to a \% level, still having high signal efficiency. Final results on this will be given in section 3.

\section{Results and outlook}

A schematic view of the rejection procedure is reported in figure 5. The cumulative number of events surviving each selection step - upward going reconstructed, $\Lambda>-5, \mathrm{R}<107 \mathrm{~m}$ and BDT $>0.35$ - for signal neutrinos and for the atmospheric muon background is reported. A possible 


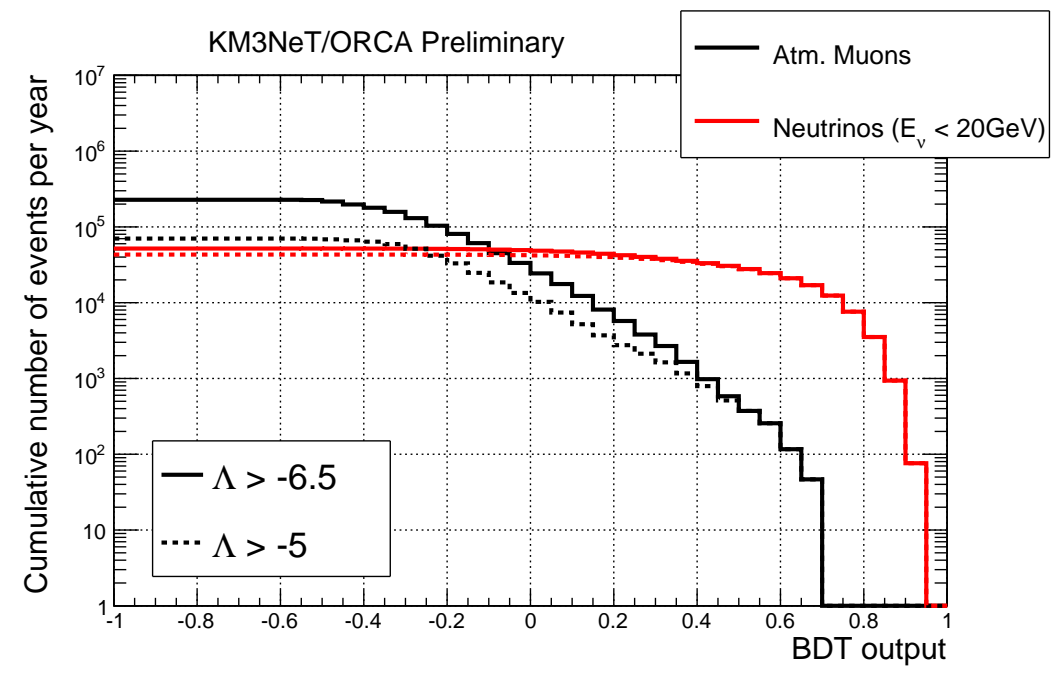

Figure 4: Output distribution of the Boosted Decision Tree technique.

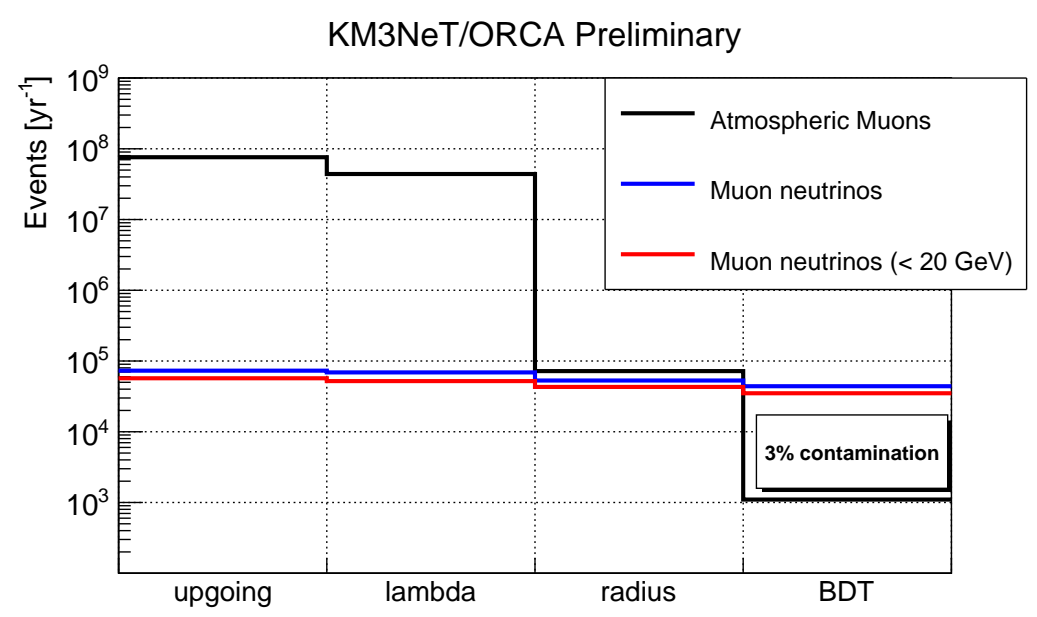

Figure 5: Step procedure for the selection of sample of low energy atmospheric neutrinos (red line) with a small contamination of atmospheric muons (black line); also shown the overall expectation for neutrino events (blue line). A 3\% contamination level is reported here, but a harder muon rejection is also possible, at expenses of the neutrino effective volume. The radius and the BDT cut are the most effective selection steps of the procedure.

contamination of the $3 \%$ in the final sample is achieved with a signal efficiency around the $65 \%$ on contained neutrino events below $20 \mathrm{GeV}$.

These results hold for the track channel, for which the procedure is optimised. Shower-like events, induced by neutral current interactions of muon and electron neutrinos and charge current interactions of electron neutrinos have not been taken into account here. An event-type classification algorithm has been developed for KM3NeT/ORCA and shows promising results in discriminating track from shower events with a multivariate technique. The same algorithm is foreseen to address also the identification and rejection of atmospheric muons within a more comprehensive 
approach. Further improvement in the rejection of wrongly reconstructed atmospheric muons are expected from this approach since measurable parameters related to shower properties can boost the topological identification of background atmospheric muons.

\section{References}

[1] www.km3net.org

[2] R. Bruijin (for the KM3NeT Collaboration), Proceedings of the $34^{\text {th }}$ ICRC, The Hague, Netherlands, ID 1157 (2015).

[3] A. Creusot (for the KM3NeT Collaboration), Proceedings of the $34^{\text {th }}$ ICRC, The Hague, Netherlands, ID 1154 (2015).

[4] S. Biagi (for the KM3NeT Collaboration), Proceedings of the $34^{\text {th }}$ ICRC, The Hague, Netherlands, ID 1164 (2015).

[5] P. Piattelli (for the KM3NeT Collaborations), Proceedings of the $34^{\text {th }}$ ICRC, The Hague, Netherlands, ID 1158 (2015).

[6] J. Brünner (for the KM3NeT Collaboration), Proceedings of the $34^{\text {th }}$ ICRC, The Hague, Netherlands, ID 1140 (2015).

[7] E.K. Akhmedov, S. Razzaque, A.Y. Smirnov, arXiv:1205.7071 (2012).

[8] L. Wolfenstein, Phys. Rev. D 17: 2369 (1978).

[9] S.P. Mikheyev and A.Y. Smirnov, Sov. J. Nucl. Phys. 42: 913 (1985).

[10] M. Jongen (for the KM3NeT Collaboration), Proceedings of the $34^{\text {th }}$ ICRC, The Hague, Netherlands, ID 1092 (2015).

[11] S. Galatà (for the KM3NeT Collaboration), Proceedings of the $34^{\text {th }}$ ICRC, The Hague, Netherlands, ID 1102 (2015).

[12] J. Hofestädt (for the KM3NeT Collaboration), Proceedings of the $34^{\text {th }}$ ICRC, The Hague, Netherlands, ID 1083 (2015).

[13] G. Carminati, M. Bazzotti, A. Margiotta, and M. Spurio. Comp. Phys. Comm. 179: 917 (2008).

[14] Y. Becherini, A. Margiotta, M. Sioli and M. Spurio, Astrop. Phys. 25: 1 (2006).

[15] A. Hoecker, P. Speckmayer, J. Stelzer, J. Therhaag, E. von Toerne, and H. Voss, TMVA - Toolkit for Multivariate Data Analysis, PoS ACAT 040 (2007), arXiv:physics/0703039. 